\title{
Pflege bei Demenz
}

\author{
Sunmed-Akademie bietet Weiterbildung nach GuKG
}

Die Betreuung und Pflege von Menschen mit Demenz und die Begleitung von Angehörigen ist für Mitarbeiter in Gesundheitseinrichtungen eine große Herausforderung. Der Umgang mit Menschen mit kognitivem Abbau, fortschreitendem Verlust der Selbstständigkeit und vielfältigen Verhaltensveränderungen erfordert ein hohes Maß an Fachwissen und die Fähigkeit zur Umsetzung in die jeweilige Pflegepraxis.

Die bisherige Ausbildung und Weiterbildung von Mitarbeitern im Pflege- und Betreuungsbereich weist hier beträchtliche Defizite auf. Die Etablierung der Weiterbildung „Pflege bei Demenz" der Sunmed-Akademie soll diese Lücken schließen.

Angesprochen sind Personen des gehobenen Gesundheits- und Krankenpflegefachdiensts sowie Pflegehelfer, die sich auf demenzgerechte, personenzentrierte Pflege und Betreuung im Rahmen ihrer beruflichen Tätigkeit spezialisieren oder ihre Kenntnisse in diesem Bereich vertiefen möchten.

Die Weiterbildung enthält unter anderem Inhalte wie Geriatrie, Gerontopsychiatrie und Neuropsychologische Diagnostik, Altenpflege, rechtliche und ethische Rahmenbedingungen, Umgang mit Verhaltensveränderungen im Rahmen der Demenz, Pflegeprozess bei Demenz und Supervision, Psychohygiene, Konfliktmanagement und Kommunikationsführung.

Ziele der Weiterbildung reichen von der Entwicklung einer positiv wertschätzenden und personenzentrierten Grundhaltung bezüglich Alter und Demenz und einer Vertiefung des Basiswissens Geriatrie über den Erwerb von Wissen über demenzspezifische psychosoziale Interventionsformen und der Fähigkeit zur reflektierten, praktischen Anwendung bis zur Fähigkeit zur psychosozialen Angehörigenarbeit sowie der Entwicklung und Vertiefung der Fertigkeiten Kommu-

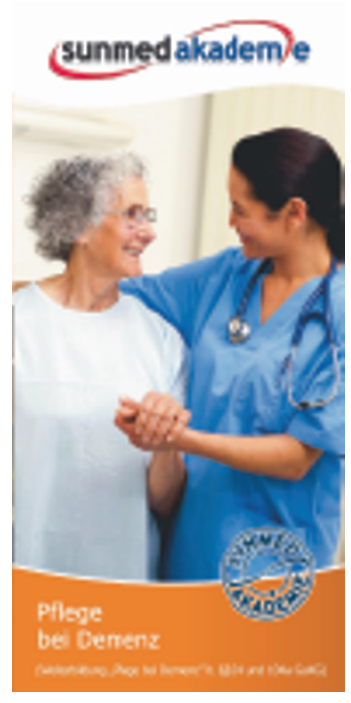

nikationsführung, Konfliktmanagement, Teamarbeit und Psychohygiene.

Die Weiterbildung umfasst ingesamt 260 Stunden, die in 160 Stunden Theorie und 100 Stunden berufsbegleitende Projektarbeit aufgeteilt sind.

Informationen: Sunmed-Akademie, Jochen-Rindt-Straße 25, 1230 Wien, Tel.: 01/699 22 99, Fax: 01/253 3033-2464, E-Mail: akademie@sunmed.at,

Internet: www.akademie.sunmed.at

\section{Gemeinsam für optimale Ernährung}

\section{Aspekte der klinischen und außerklinischen Patientenversorgung}

Der Bereich Ernährung wird bei der Behandlung verschiedener Erkrankungen oft unterschätzt. Dabei kann eine optimal angepasste Ernährungstherapie Komplikationen mindern, Verweildauern verkürzen, die Genesung beschleunigen und Lebensqualität steigern.

Moderne Strategien rückt der Themenblock „Ernährung“ des MEDCARE-Fachkongresses vom 30. September bis 1 . Oktober 2015 in Leipzig in den Mittelpunkt. Falldiskussionen und Workshops in Zusammenarbeit mit renommierten Fachgesellschaften beleuchten unter anderem Ernährungsaspekte in der Krebstherapie sowie die Bedeutung der Ernährung für die Vermeidung nosokomialer Infektionen.

„Eine bestmögliche Ernährungstherapie erfordert die interdisziplinäre, interprofessionelle Zusammenarbeit von
Pflege, Ärzten sowie Ernährungs- und Diätassistenten“, erklärt Prof. Dr. Arved Weimann, Chefarzt der Klinik für Allgemein- und Viszeralchirurgie des Klinikums St. Georg gGmbH in Leipzig. „Ich werde besonders auch auf den Zusammenhang zwischen Ernährung und Anfälligkeit für nosokomiale Infektionen hinweisen", betont Weimann. Anhand von Fallbeispielen wird er sich auch speziellen Ernährungskonzepten für Tumorpatienten widmen, darunter Standards für den ambulanten und stationären Umgang mit künstlicher Ernährung.

\section{Ernährungsmedizin fest etablieren}

„Zurzeit wird die Ernährungsmedizin nicht richtig ernst genommen. So erfassen weniger als zehn Prozent der chirurgischen Kliniken in Deutschland bei der
Patientenaufnahme den Ernährungsstatus“, schätzt Weimann. Dabei seien Ernährungsdefizite unmittelbar verbunden mit der Empfänglichkeit des Körpers für Infektionen. „Auf die Risiken von Mangel- und Fehlernährung achten wir im klinischen Alltag immer noch zu wenig. Es muss uns besser gelingen, die Ernährungsmedizin fest zu etablieren sowie tragfähige und sektorenübergreifende Strukturen zu schaffen, die im Krankenhaus und darüber hinaus in der ambulanten Betreuung sowie in Pflegeeinrichtungen greifen." Gerade für alte Menschen und Menschen in Pflegeheimen seien weiterführende Ernährungskonzepte wesentlich, konstatiert Weimann.

Quelle: Presseinformation Medizinische Messen Informationen: www.medcare-leipzig.de/ veranstaltungsprogramm 